\title{
A Deep Esophageal Injury from a Lithium Battery: A Case Report
}

\author{
Arrigo Vittorio Barabino ${ }^{1 *}$, Gian Michele Magnano ${ }^{2}$, P. Gandullia ${ }^{1}$ and M. Leoni ${ }^{1}$ \\ ${ }^{1}$ Gastroenterology and Digestive Endoscopy Unit, Istituto G. Gaslini (IGG), Genoa, Italy \\ ${ }^{2}$ Radiology Department, Istituto G. Gaslini (IGG), Genoa, Italy
}

\begin{abstract}
*Corresponding author: Arrigo Vittorio Barabino, MD, Head of Gastroenterology and Digestive Endoscopy Unit, G. Gaslini Institute, Via G. Gaslini 5, 16148, Genoa, Italy; Tel: +3901056362771; Fax: +39010383688; E mail: arrigobarabino@gaslini.org or abarab@ gmail.com
\end{abstract}

Article Type: Case Report, Submission Date: 21 December 2015, Accepted Date: 11 January 2016, Published Date: 14 March 2016.

Citation: Arrigo Vittorio Barabino, Gian Michele Magnano, P. Gandullia and M. Leoni (2016) A Deep Esophageal Injury from a Lithium Battery: A Case Report. J.Paedi.Care.Inol 1(1): 6-8. doi: https://doi.org/10.24218/jpci.2016.02.

Copyright: @ 2016 Arrigo Vittorio Barabino, et al. This is an open-access article distributed under the terms of the Creative Commons Attribution License, which permits unrestricted use, distribution, and reproduction in any medium, provided the original author and source are credited.

\section{Abstract}

Context: Over the last ten years the ingestion of disk batteries and its serious consequences have been increasing. The severity of injury is related to a growing diffusion of the new lithium battery that may cause catastrophic damages when lodged in the esophagus in children.

Case report: A five-year-old boypresented to the Emergency Department of our tertiary pediatric Institute for a lithium battery lodged in the mid esophagus. Emergent esophagoscopy revealed a severe deep, mild bleeding ulceration of the wall in which the battery was partially wedged. The investigation was stopped and on-call cardio-vascular surgeon started left thoracotomy to exclude damages of the main vessels. With the thorax open, the endoscopy was repeated and a directional relationship between the battery and the aorta was excluded by means of transillumination. The cell, a CR2032 lithium battery, was then removed. Central line parenteral nutrition, i.v. omeprazole plus antibiotics were started with a drainage tube left in the chest. During the follow-up the child undergone several chest X-rays with the suspicion of esophageal perforation. Angio-TC done on day 7 showed air into the thickened esophageal wall and in the mediastinum with severe peri-aortic edema without lesion of the vessel. MRi performed on day 21 showed only a persistent thickening of the esophageal wall. On day 28 an esophagogram was normal and the child was discharged asymptomatic. Two months later the investigation was repeated resulting entirely normal.

Conclusions: Treatment of disk battery ingestion requires a multidisciplinary approach that can be implemented only in a tertiary pediatric hospital. Surgery can play an important role. Severe complications can occur several days after battery removal.

Keywords: Lithium battery ingestion, Aorto-esophageal fistula, Esophageal injury, Children.

\section{Introduction}

The ingestion of disk battery may cause catastrophic complica- tions, and 44 deaths in children have been reported worldwide so far [1]. Sixty-six \% of them were due to a fistula between esophagus and aorta or other major vessels. In order to stop the continuing rise in life-threatening injuries several multi-faceted approaches have been proposed [2-7].

The severity of injury depends on cell type, size, voltage, location and time of tight contact with the mucosa. The main lesion mechanism is an electrical generation of hydroxide ions at the negative pole causing an alkaline burn proportional to the battery voltage. Lithium batteries are larger $(>20 \mathrm{~mm})$, flatter and have an higher voltage (3V) than the alkaline (nickel, zinc, mercury or silver oxide) disk batteries $(1.5 \mathrm{~V})$. In small children the ingestion of such cells increases the risk of esophageal lodgment and significant tissue damage in just two hours $[2,3]$. The electric injury occurs when both the poles are in strict contact with the mucosae as it happens in the esophagus with all kind of battery, the exhausted included [2]. This process is much less possible if the cell lies in a big cavity like the stomach or rapidly transits along the intestine. The alkaline battery in the stomach can also be dangerous for the leakage of its toxic content due to the acid presence.

Here we describe a child with alithium battery lodged into the esophagus seen in our a tertiary pediatric Institute. The peculiarity of the case is that the child required urgent thoracotomy to exclude aorta involvement suggested by severe ulcerations of the esophagus seen at endoscopy. The outcome was complicated by suspected esophageal perforation fully recovered with conservative treatment.

\section{Case report}

Case report 2: A 5-year-old boy was admitted on September, 2012 for three day history of epigastric pain persisting despite oral omeprazole. The mother reported possible ingestion of a coin. A plain chest and abdomen X-ray showed a lithium battery lodged in mid-oesophagus (Figure 1). No history of previous sentinel bleed was reported and physical examination was unremarkable. Blood tests were normal. The patient, monitored by an anesthesiologist, was immediately transferred to the 


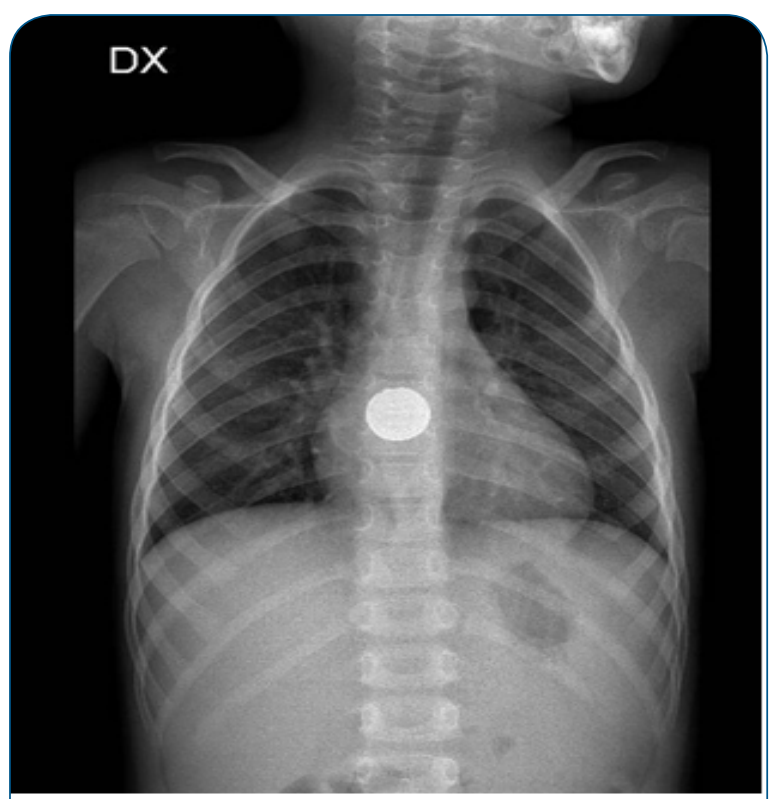

Figure 1: Lithium battery lodged in the mid esophagus

operating room where the GI endoscopist was ready to remove the battery. Oesophagoscopy (GIF-180 video gastroscope, Olympus, Milan) revealed a severe, deep, mildly bleeding ulceration of the oesophageal wall in which the battery was partially wedged. Oesophagoscopy was stopped, while the anesthesia was continued. Within 25 minutes, the on-call cardiovascular (CV) surgeon started left thoracotomy to exclude damages of the aorta. With the thorax open, an intra-operative assisted endoscopy was done, and a direct contact between the battery and the aorta was excluded by means of transillumination. The battery, a CR2032 lithium battery, was pushed into the stomach and then removed. Central line parenteral nutrition, open naso-gastric tube, i.v. omeprazole, and antibiotics were started. During the follow-up the child undergone several chest X-rays with the suspicion of esophageal perforation. Angio-TC done on day 7 showed air in the thickened esophageal wall and in the mediastinum, with severe peri-aortic edema without lesion of the vessel (Figure 2). MRi performed on day 21 showed only a persistent thickening of the esophageal wall. On day 28 an esophagogram was normal

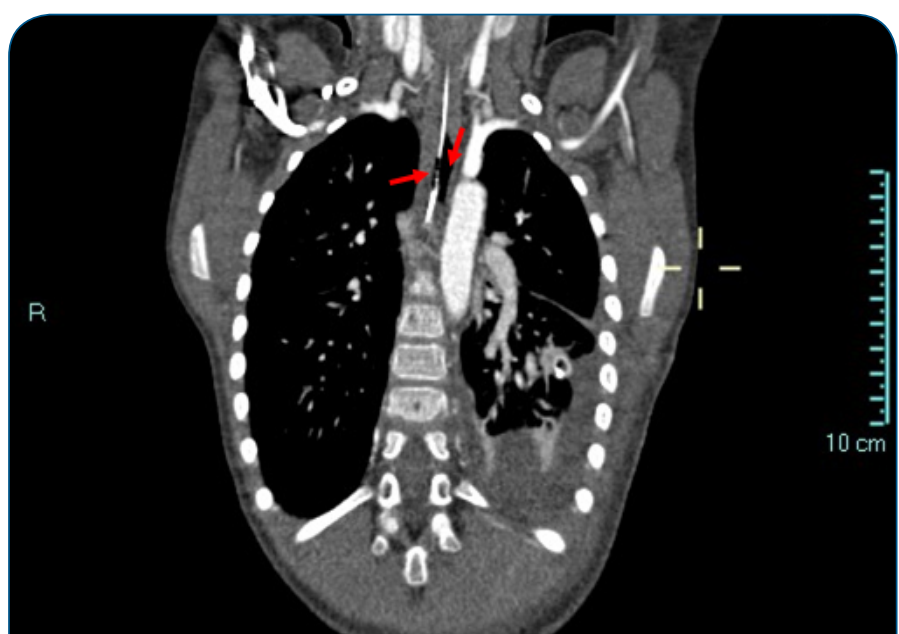

Figure 2: Angio CT showingair within the esophageal wall and into mediastium (red arrows) and the child was discharged asymptomatic. Two months later the investigation was repeated resulting entirely normal.

\section{Discussion}

Not witnessed ingestion of a disk battery have significant implications on primary care setting [8]. Clinical presentation may be extremely variable and difficult to recognize. The child with a disk battery located in the GI tract can be symptom-free or to present typical symptoms such as drooling, dysphagia, vomiting, chest pain or dyspnea. Atypical symptoms are fever, abdominal pain, irritability and feeding refusal. Sudden fatal exsanguination for a fistula between oesophagus or other major vessels of the mediastinum can also be a dramatic presentation $[4,5]$. Other reported complications are: tracheo-esophageal fistula, laryngeal or esophageal stenosis, esophageal perforation, vocal cord paralysis, tracheomalacia, aspiration pmeumonia, empyema, lung abscess and spondylodiscitis [4]. Complications can be delayed, as the mucosal lesions could worsen after cell removal. Plain chest and abdomen X-ray investigations have a primary role to make the diagnosis and to locate the battery, that can be recognized by the presence of the double ring or "halo" effect.Urgent endoscopic removal of the disk battery depends on the clinical presentation.

In 2011 it was documented that a "sentinel bleed", such as an isolated hematemesis or melena occurring hours or days before a fatal hemorrage, might be another atypical presenting symptom [5]. Exsanguination may occur with the battery still in the GI tract or after its removal. The interval between battery removal and fatal hemorrhage ranges between 2 and 28 days [1,2]. In stable patients with"sentinel bleeds" there is a time window that allows a successful surgical intervention, as suggested by the protocol developed by Brumbaugh and coworkers [5].

In this case the single symptom related to lithium battery stuck in the esophagus was epigastric pain. X-ray was able to correctly differentiate it from a coin. According to the Brumbaugh's protocol [5] the child should have been managed by an emergent gastroscopy to immediately remove the cell. Because the severe esophageal lesion was suggestive for a possible involvement of the aorta or other major vessels of the mediastinum, the cell was not touched. Despite the lack of a previous "sentinel bleed", we wished to avoid the "activation" of an underling vascular fistula with an immediate fatal consequence. At that time the battery could act as a plug of a vessel lesion preventing a major bleeding to occur. According to that the Brumbaugh guideline [5] was not followed, and we chose to exclude vessels damage by thoracotomy. This case underlies the crucial role of the endoscopy in this clinical presentation because the severity of the lesions indicates next operating steps, andinduced us to modify the multidisciplinary approach to DB ingestion in children [7] .

As reported in the literature $[3,4,9]$ several days after the cell removal a suspected esophageal perforation with a periaortic severe edema occurred highlighting the potential late consequences of the $\mathrm{DB}$ ingestion, requiring a close clinical follow-up by means of traditional radiology, CT scan, MRi or repeating endoscopy. 
Citation: Arrigo Vittorio Barabino, Gian Michele Magnano, P. Gandullia and M. Leoni (2016) A Deep Esophageal Injury from a Lithium Battery: A Case Report. J.Paedi.Care.Inol 1(1): 6-8. doi: https://doi.org/10.24218/jpci.2016.02.

\section{References}

1. National Capital Poison Center. Available from : http://www.poison. org/battery/fatalcases.asp

2. Jatana KR, Litovitz T, Reilly JS, Koltaie PJ, Riderf G, Jacobs IN. Pediatric button battery injuries: 2013 task force update. International Journal of Pediatric Otorhinolaryngology. 2013; 77: 1392-99.

3. Litovitz T, Whitaker N, Clark L. Preventing battery ingestions: an analysis of 8648 cases. Pediatrics.2010; $125:$ 1178-83.

4. Litovitz T, Whitaker N, Clark L, White NC, Marsolek M. Emerging battery ingestion hazard: clinical implications. Pediatrics. 2010; 125: 1168-77.

5. Brumbaugh DE, Colson SB, Sandoval JA, Karrer FM, Bealer JF, Litovitz $\mathrm{T}$, et al. Management of button battery-inducedhemorrhage in children. J Pediatr Gastroenterol Nutr. 2011; 52(5): 585-9. doi: 10.1097/ MPG.0b013e3181f98916.
6. Rossi A, Barabino A, Vignola S, Bini M. Lithium disk battery: the silent killer. J Gastroenterol Hepatol Res. 2015; 4: 1624-34.

7. Kramer RE, Lerner GD, Lin T, Manfredi M, Shah M, Stephen TC, et al. Management of ingested foreign bodies in children: a clinical report of the NASPGHAN Endoscopy Committee. J Pediatr Gastroenterol Nutr. 2015; 60: 562-74.

8. Shepherd M, Hamill JK, Barker RJ. Buttonbattery injury in children - a primary care issue? J Prim Health Care. 2014; 6: 69-72.

9. Soccorso G, Grossman O, Martinelli M, Marven SS, Patel K, Thomson $\mathrm{M}$, et al. $20 \mathrm{~mm}$ lithium battery causing an oesophageal perforation in a toddler: lesson in diagnosis and treatment. Arch Dis Child. 2012; 97: 746-47. 